\title{
Discussion on the Function of Cross-line Graph Edited Software for Urban Rail Transit
}

\author{
Teng ChunGuang ${ }^{1}$, Hao Qingsheng ${ }^{2}$, Liang Hongyu ${ }^{3}$
}

(Beijing Traffic Control Technology Co., Ltd. Beijing 100070)

Key Words: cross-line; train graph; general graph; composition graph, single graph

Abstract: This paper discusses the working diagram function of city rail traffic across the line train graph diagram of software, as well as for the cross line working diagram function realization method, put forward the general line and cross mapping strategy, summed up the general layout diagram and sequence diagram is divided into general compilation of the pros and cons of each order. That there are relatively large differences across the line diagram compilation of city rail traffic and ordinary working diagram, in the train graph diagram software function is to make a relatively large change in the use of cross line train graph diagram software cross line diagram, sequence according to the business process and habits of each line to the operator the general decided to compile the map.

\section{Overview}

In large cities with a subway, there are usually a number of subway or light rail lines. Usually the lines are completely closed and independent, and they are independent and do not relate to other lines. However, with the increase of the carrying capacity of the urban rail transit and the increase of the number of trips, the closed single line operation mode can not meet the current travel requirements of the line resource utilization efficiency. It is mainly shown in the following points:

(1) Transfer times: more passengers from origin to destination may span multiple lines, to achieve in the current mode only through the transfer, transfer, increase the retention in the metro line within the time, reduces the efficiency of subway operation, increase the passenger travel time cost.

(2) The demand for spare cars is large: in every line, in order to ensure the normal operation of the subway system, we need to reserve cars at any time to replace the failed vehicle on-line, which brings the operation cost improvement.

As the train runs across the line, the above problems can be solved in varying degrees. In order to improve the utilization of line resources, the concept of cross line operation has been put forward, and it has been paid attention to by the industry. The key to the cross line operation lies in the rational layout of the cross line operation diagram and the coordination of the urban rail traffic organization. Therefore, the traditional mapping software is also facing innovation. This paper focuses on the new problems faced by the mapping software and the new ideas to solve the problem.

\section{Cross line mapping strategy}

\section{Traditional single line operation diagram}

Train diagram is the comprehensive plan of urban rail traffic organization work, and it is the basis of subway and light rail traffic organization. The train diagram is not only the combination of each station, 
along the line, power supply, communication technology, vehicle signal equipment into a unified whole, and all traffic related departments and units are organized strictly according to certain procedures to work everything in good order and well arranged, so as to ensure train safety and punctuality.

Train diagram is a graphic form of coordinate principle to express train operation. The train diagram shows the time with the transverse coordinates, the longitudinal coordinates represent the distance, the horizontal line represents the position of the center line of each station, and the slash is called the train line. The next picture is a train diagram.

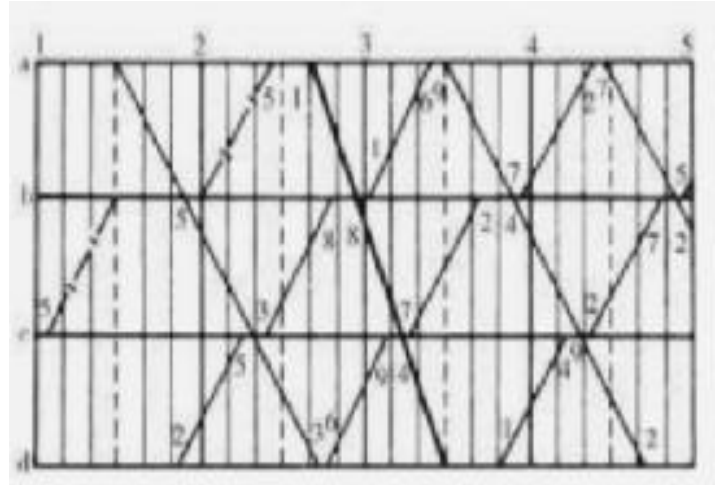

Fig. 1 Train graph

\section{Cross line mapping strategy}

Cross line operation diagram is a traffic plan across multiple lines. Then it is necessary to form a diagram of two aspects to represent the entire cross line operation diagram. On the one hand, it is a general drawing, which can describe the running time of trains running across multiple lines throughout the whole process. On the other hand, it is a sub graph, which describes the operation of all the vehicles on each line, and corresponds to the original operation diagram of a single line. From these two perspectives, the operation plan of all trains in the road network can be described.

In compiling the map, the above two aspects of seamless combination is a difficult point, because the cross line is part of the train, but in the current application scenario, it is very likely that a small number of trains are running, most of them are still running in the line. The inconsistency of the running section of a cross - line vehicle and a non - Cross - line vehicle greatly interferes with the compilation of the operation diagram. It is mainly shown that the trans line vehicle is difficult to turn back in a short time, rather than the trans line vehicle will soon turn back to run. When the trans line vehicle is adjusted at the crossing line time point, it will directly affect the adjustment of the two lines.

Due to the above reasons, when drawing up the cross line operation diagram, there should be a sequential order between the general layout and the line map, either after the general layout or the general plan. It is difficult to connect seamlessly if it is compiled at the same time. Each of the two sequences has its own advantages and disadvantages. First, the general layout and the sub map. The advantage is that the general layout can not make any other factors, so that we can achieve the goal of the general layout first. The drawback is that only after the general plan is completed can we make the subsections of all the lines. Once the general layout is modified, it is very likely that the drawings will need to be re painted. First, the general plan is partite after the map. The advantage is that the partite graph can be consistent with the previous single line drawing. The modification of the general layout will not affect the partite graph. The drawback is that all the elements in the map should be considered when the general graph achieves the goal, and the general layout will be changed accordingly when the map is modified. This paper focuses on the development strategy of the first general map. 


\section{cross line drawing steps}

\section{Identifying the basic elements}

Although the train diagram has a variety of different types, it is always made up of some basic elements. Therefore, before the general train diagram is compiled, the elements of the train diagram must be determined first. The basic elements of the train diagram including train running time, stop time, time, reentry running cross the road and train out of storage and time, all day long operation time, the number of vehicles using the foreign and Gaoping low peak hours, the train track interval, during the operation of train line servicing etc.. The above elements are far from enough in the compilation of the cross line operation diagram, and the cross line related elements need to be added. As this article is across the line, the following sub item illustrates the cross line elements in the mapping function of the cross line running graph software.

\section{Set up a crossing line}

Before drawing up the general drawing of cross line operation diagram, we must first set up spanning lines, and trains that usually cross the same lines may not be consistent on each line's stop. Therefore, one of the elements of the trans route is crossing the line's combination. We set up and store the crosses in the software so that we can easily select the line combinations that we often use.

\section{Making cross line total diagram constraints}

Cross the line drawing in general, to determine the cross line and display across the line in the station parking, no parking across the line in the station is not necessary to display, is conducive to drawing general use templates in the drawing layout before, can use the general protocol, and improve the efficiency of drawing layout, reduce the error may.

\section{Create a contact line}

When crossing the line, there is a necessary element, that is, how to cross the past between the two lines and where to go across. This is the tie line. When we use the cross line function, we first create the tie line in the software, and specify which line should be used when crossing the line. Secondly, set the parameters of each contact line, such as: setting the length of the contact line, passing the time, running direction, and so on.

\section{Compilation of general drawings}

Preparation of general layout, mainly used to determine after the train entered the cross the line of time, including train all lines across the drawing in the coordinate system, with a depot train departure route may be different, if these routes are cross line situation, then in the same total figure is at the same time to display. Therefore, a general map shows only one driving route in principle. In accordance with the strategy of the following sub graph, the method of drawing up the total map and the sub graph is explained.

Before drawing up the general plan, we must first set up the station in the drawing coordinate system according to the driving route, and take the stations on all the routes in the driving route as the stations on a line. After setting up, the coordinate system of the general map is saved, so it is convenient to use the general diagram of the same route later.

Compiling a general map according to the compilation of a line. After the completion of the compilation, the mapping software can automatically form the timetable across the lines to prepare each sub map. For cross - line time, the software is shown on the general diagram in accordance with the running time of the contact line.

\section{Compiling sub graph}

Map compilation, first select the line and general layout, software generation in this line number, and 
displayed on the map, and then run the map on the basis of the upper line, in the process of drawing the map, and never change in the line number.

After the completion of the painting, the train runs according to the sub map.

In the preparation of cross line diagram, relates to the general and mapping software, if the map and map is not a manufacturer, which will bring mutual recognition diagram, the problem of mutual use, then you need to form a standard format specification diagram.

In many city, there may be the line operator is not the same, so that mutual cooperation is a need to consider the issue of implementation of the software, is to confirm the time and location for each table, cross the line, and trains, in the early stage of cross line operations, can use the phone or mail confirmation way, in the mature period, it can be directly used in coordination with the synergic relationship protocol software.

\section{Summary}

There is a great difference between the cross line operation diagram of urban rail transit and the general operation diagram, and it is necessary to take into account the situation of each line. The main difference is to use two forms of operation diagram at the same time, one is general layout, the other is sub map, so that we can fully describe the planning and operation of trains. In order to adapt to this change, timetabling software also need to do some big changes, adding compilation of two forms of the diagram, and combine these two ways, do some preprocessing, such as information, adding line information, contact line information, and the general format, for example, cross line map for standard interface. It makes the cross - line mapping form a complete function.

\section{Acknowledgements}

Beijing Science and Technology Commission project "The research and demonstration of Beijing subway network operation train graph and vehicle signal equipment (D161100004816001)"provides funding.

\section{Reference}

[1] [1] Chen Xingzhao, Yang Changxiu, Li Hang. Feasibility Study of Cross-line Operation of Urban Rail Transit[J]. Smart City, 2016, 2(04): 250-251

[2] [2] Liu Jiali. Discussion on coordination issues of train cross-line operation [J]. New West, 2013(2): 93-94

[3] [3] CAI Changjun, YAO Enjian, ZHANG Yongsheng. Prediction of traffic distribution among urban rail stations based on AFC data by Liu Shasha, China Railway Science, 001-4632 (2015) 01-0126-07

[4] LIN Zhan, JIANG Ming qing, LIU Jian feng , SI Bing feng, Improved Logit M odel and M ethod for Urban Rail Transit Network Assignment, Journal of Transportation Systems Engineering and Information Technology, 001-4632(2015)01-0126-07

[5] BAI Li,Urban Rail Transit Norm al and Abnorm al Short. term Passenger Flow Forecasting M ethod, Journal of Transportation Systems Engineering and Information Technology, 1009-6744(2017)01-0127-09

[6] Zhang Yi. Effects of Cross-line Trains on Passenger Dedicated Lines and Existing Lines' Capacity and 
Division of Labor[J].Zheng Tie Science and Technology Communications,2006(1)

[7] Lin Ruihua. Rail Transit Passenger Flow Forecasting Based on Time Series Analysis, Modern Economic Information, 1001-828X (2013) 11-0370-01

[8] Xie Dapeng, Ye Yuling, Ji Ling. Planning concept of urban rail transit network system in metropolitan area[J]. Urban Rail Transit Research. 2006(6): 22

[9] Zhang Mei, Guo Fu'an. Research on related technologies of passenger dedicated railway lines [J]. Chinese Railways, 2007 (12) 21-25

[10] Beijing Municipal Planning Commission. GB50157-2013 Metro Design Specification [S]. Beijing, 2014.3 